\title{
Using Geometry Invariants for Camera Response Function Estimation
}

\author{
Tian-Tsong Ng, Shih-Fu Chang \\ Department of Electrical Engineering \\ Columbia University \\ New York, NY 10027 \\ \{ttng, sfchang\}@ee.columbia.edu
}

\author{
Mao-Pei Tsui \\ Department of Mathematics \\ University of Toledo \\ Toledo, $\mathrm{OH} 43606$ \\ mao-pei.tsui@utoledo.edu
}

\begin{abstract}
In this paper, we present a new single-image camera response function (CRF) estimation method using geometry invariants (GI). We derive mathematical properties and geometric interpretation for GI, which lend insight to addressing various algorithm implementation issues in a principled way. In contrast to the previous single-image CRF estimation methods, our method provides a constraint equation for selecting the potential target data points. Comparing to the prior work, our experiment is conducted over more extensive data and our method is flexible in that its estimation accuracy and stability can be improved whenever more than one image is available. The geometry invariance theory is novel and may be of wide interest.
\end{abstract}

\section{Introduction}

Camera response function (CRF) maps image irradiance (light energy incident on image sensors) to image intensity (output of a camera). In practice, the mapping is a collective effect of various camera internal operations and noise. The capability to estimate CRF is important, as various photometric computer vision algorithms, such as shape from shading and photometric stereo, require image irradiance. If $\mathrm{CRF}$ can be estimated, image intensity can be transformed to image irradiance. Furthermore, a CRF can be thought as a natural watermark and used for image authentication.

Assuming that CRF is spatially uniform in an image, CRF can be estimated from three types of inputs: a set of same-scene images with different but known exposures [8, $2,10]$, a single RGB color image [6], or a grayscale image converted from a RGB color image [7]. Estimating CRF from a single image is an under-constraint problem and requires a physical assumption on image irradiance, e.g., the distribution of the image irradiance value at a local edge region is assumed to be uniform [7]. Unfortunately, for all previous single-image CRF estimation methods, there is no

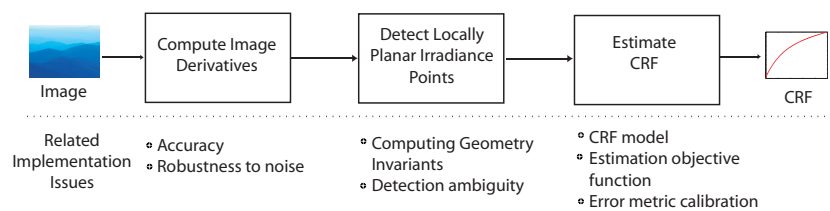

Figure 1. The computational steps for our CRF estimation method and their related implementation issues.

principled mechanism to identify image regions which satisfy their assumptions, as verifying assumptions in the unknown image irradiance domain is non-trivial.

In this work, we propose a theoretical-based CRF estimation method using geometry invariants (GI), which provides a constraint equation to identify the potential locally planar irradiance points (LPIP) used in CRF estimation. The locally planar region in image irradiance can be found on the ramp edges in an image. Our method consists of three main computational steps, i.e., computing image derivatives, detecting LPIP, and CRF estimation, as shown in Fig. 1. In Fig. 1, we also show the related implementation issues. Our experiments are conducted on 5 models of camera (from 4 different manufacturers) compared to three in [6] and two in [7]. Apart from single-image CRF estimation, our method can achieve a better estimation accuracy and stability when multiple images from the same camera are available.

In this paper, we describe the prior work on CRF estimation in Sec. 2. In Sec. 3, we present the theoretical aspect of the CRF estimation algorithm. In Sec. 4, we describe a method to overcome an ambiguity in our method, and in Sec. 5, we describe CRF estimation through curve fitting. In Sec. 6, we describe the implementation aspect of our algorithm. Finally, we show our experiments in Sec. 7 and conclude with Sec. 8.

\section{Prior Work on CRF Estimation}

CRF's can be manually estimated by photographing a Macbeth chart with known-reflectance patches, under uniform illumination. CRF's recovered using a Macbeth chart 
are considered reliable and often accepted as a ground truth for evaluating other CRF estimation techniques [5, 6, 7].

On the other hand, automatic CRF estimation methods relies on assumptions defined in the irradiance domain. As CRF transformation of image irradiance results in a deviation of the assumptions, CRF can be estimated as a function $f$ which best restores the assumptions. For methods that estimate CRF using multiple same-scene images [8, 2, 10], the exposure ratio among the images provides a relationship between the irradiance images and the CRF is estimated by a function $f$ that restores this relationship from the intensity image sequence.

In an earlier work [3], a CRF estimation method for a single-channel image is proposed by assuming that nonlinear transformation of image irradiance by a gamma curve, $f(r)=r^{\gamma}$, introduces correlated harmonics, which can be measured by the magnitude of biocoherence (thirdorder moment spectra). However, the method is limited to the use of the gamma curve CRF model, which is known to be insufficient for real-world CRF's. In [6], a CRF estimation method using a single RGB-color image by assuming linearly blended edge pixels (between two homogenous regions with distinct irradiance values). When the linear blending assumption holds across the RGB color channels, it can be shown that the image irradiance values at the edge and the adjacent homogenous regions will be colinear in the RGB color space. Additionally, the assumption that the image irradiance values in an image edge region form a uniform distribution was employed to estimate CRF from a grayscale image [7]. However, the method is only demonstrated on grayscale images converted from RGB color images instead of single-color channel images [7].

\section{Theoretical Aspects of the Algorithm}

In this paper, we use $r(x, y)$ and $R(x, y)$ respectively for image irradiance and image intensity. CRF is denoted either by $R=f(r)$, or $r=g(R)$. The 1 st-order derivatives $\frac{\partial R}{\partial x}$ and $\frac{d f(r)}{d r}$ are respectively denoted as $R_{x}$ and $f^{\prime}(r)$, with similar convention for higher-order derivatives.

\subsection{Geometry Invariants}

Given $R(x, y)=f(r(x, y))$, we take the 1st-order partial derivatives of $R(x, y)$ and by the chain rule we obtain:

$$
D R(x, y)=\left(\begin{array}{ll}
R_{x} & R_{y}
\end{array}\right)=f^{\prime}(r)\left(\begin{array}{ll}
r_{x} & r_{y}
\end{array}\right)
$$

Note that $R_{x}$ is the product of two factors; the first factor $f^{\prime}(r)$ is purely related to CRF while the second factor $r_{x}$ is purely related to the geometry of image irradiance. GI can be derived if the second factor, the effect of image geometry, can be removed. Hence, the resulting GI is only dependent on CRF $f$ but not the geometry of image irradiance.
It is non-trivial to eliminate the geometry effect of an arbitrary function $r(x, y)$. However, a function can be locally approximated by its Taylor expansion, which decomposes the local geometry into polynomials. The 1st and 2nd-order polynomials are respectively planes and quadratic functions. We can define the 1 st-order GI $\left(\mathcal{G}_{1}\right)$ as quantities that are invariant to the class of planar surfaces:

$$
\{r(x, y): r(x, y)=a x+b y+c, \quad a, b, c \in \mathbb{R}\}
$$

For planes, we have $r_{x x}=r_{x y}=r_{y y}=0$, and the 2 ndorder partial derivatives of $R=f(r)$ are given by Eq. 3

$D^{2} R(x, y)=\left(\begin{array}{ll}R_{x x} & R_{x y} \\ R_{y x} & R_{y y}\end{array}\right)=f^{\prime \prime}(r)\left(\begin{array}{cc}r_{x}^{2} & r_{x} r_{y} \\ r_{x} r_{y} & r_{y}^{2}\end{array}\right)$

Then, by taking the ratio of Eq. 3 over Eq. 1, we obtain $\mathcal{G}_{1}$ :

$$
\frac{R_{x x}}{R_{x}^{2}}=\frac{R_{y y}}{R_{y}^{2}}=\frac{R_{x y}}{R_{x} R_{y}}=\frac{f^{\prime \prime}\left(f^{-1}(R)\right)}{\left(f^{\prime}\left(f^{-1}(R)\right)\right)^{2}}=\mathcal{G}_{1}(R)
$$

Note that, $\mathcal{G}_{1}$, as a function over $R$, depends only on the derivatives of $f$, but not the 1st-order geometry of image irradiance. Such strict dependence relation on $f$ will be explored in this paper to estimate $f$. We will refer to the first two equality relations in Eq. 4 as derivative equality constraints in the rest of the paper, as they imply certain important geometric properties.

\subsection{General Properties of $\mathcal{G}_{1}$}

In this section, we present two general properties of $\mathcal{G}_{1}$, related to the CRF estimation algorithm. Further properties will be described in the later sections.

Property 1 (Affine Transformation Invariance). The functional $\mathcal{G}_{1}$ is preserved, as the 3-D graph of a planar irradiance $S=[x, y, r=a x+b y+c]^{T}$ undergoes affine transformation:

$$
\text { If } A S+B \rightarrow S^{\prime} \text { then } \mathcal{G}_{1}(f(r)) \rightarrow \mathcal{G}_{1}\left(f\left(r^{\prime}\right)\right)
$$

where $A$ is a $3 \times 3$ linear transformation matrix, with $|A| \neq$ $0, B$ is a $3 \times 1$ translation vector, and $S^{\prime}=\left[x^{\prime}, y^{\prime}, r^{\prime}\right]^{T}$.

Note that, despite the value change from $r$ to $r^{\prime}$, the underlying function $\mathcal{G}_{1}$ remains the same in Eq 5. The affine transformation maps a plane to another plane, but as $\mathcal{G}_{1}$ is independent of the plane geometry, so it does not change under the transformation. Affine transformation includes rotation, scaling, and translation and is usually imposed on image irradiance when it undergoes white-balancing and contrast adjustment [13]. Therefore, with Property $1, \mathcal{G}_{1}$ is a natural instrument for CRF estimation.

A special case of affine transformation is the rotation of the graph $S=[x, y, r]^{T}$ in the $(x, y)$ plane at a point $p$ 


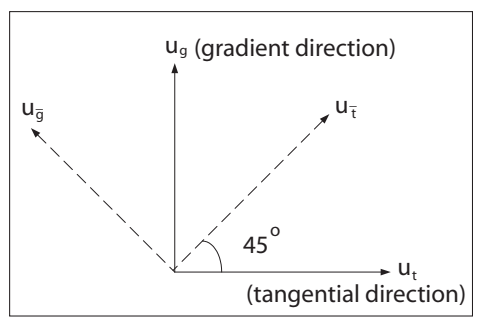

Figure 2. The gauge coordinates for computing $\mathcal{G}_{1}$.

where $r(p)=r_{p}$. In this case, we have $r_{p}=r_{p}^{\prime}$ and the value of $\mathcal{G}_{1}$ is preserved:

$$
R_{2 \times 2}\left(\begin{array}{l}
x \\
y
\end{array}\right) \rightarrow\left(\begin{array}{l}
x^{\prime} \\
y^{\prime}
\end{array}\right) \Rightarrow \mathcal{G}_{1}\left(f\left(r_{p}\right)\right)=\mathcal{G}_{1}\left(f\left(r_{p}^{\prime}\right)\right)
$$

where $R_{2 \times 2}$ is a $2 \times 2$ rotation matrix. As rotation of image irradiance is equivalent to rotation of the local coordinate frame, then Property 1 also implies that the value of $\mathcal{G}_{1}$ is preserved under local coordinate frame rotation.

Property 2 (Integral Solution to $\mathrm{CRF}$ ). The partial differential equation $(P D E)$ in Eq. 4 can be solved by an integral function of $\mathcal{G}_{1}$ :

$$
f^{-1}(R)=\int \exp \left(-\int \mathcal{G}_{1}(R) d R\right) d R
$$

Despite the above analytical solution for CRF, its feasibility is in practice deterred by detection ambiguity (Subsec. 3.3) and the solution will be approximated by a computational method described later.

\subsection{Detection of Locally Planar Irradiance Points}

We have shown that the derivative equality constraint of Eq. 4 is satisfied for every LPIP. Therefore, we may use this constraint to detect candidate points for LPIP in an image. We will also show later that a more general type of surface with linear isophote also satisfies the equality constraint. We call such inability to uniquely detect LPIP detection ambiguity, which will be addressed in Sec. 4 .

Although Property 1 implies in theory that there is not a preferred Cartesian coordinate frame for computing $\mathcal{G}_{1}$ because rotation in the local coordinate frame does not change the value of $\mathcal{G}_{1}$. In practice, it is not a good idea to simply compute the $\mathcal{G}_{1}$ on the original $(x, y)$ coordinate frame of an image. The reason is that, for example, when isophote (i.e., constant intensity curve) coincides with the $x$-axis, singularity happens for $\mathcal{G}_{1}(R)=\frac{R_{x x}}{R_{x}^{2}}$, as $R_{x}=0$ along an isophote. For computation, we introduce two first-order gauge coordinate frame, which are locally dictated by the intrinsic property of the image function: $\left(u_{t}, u_{g}\right)$ and $\left(u_{\bar{t}}, u_{\bar{g}}\right)$-coordinate frames (see Fig. 2). $u_{t}$ and $u_{g}$ are respectively the local tangential and gradient direction of an image function, and $\left(u_{\bar{t}}, u_{\bar{g}}\right)$-frame is rotated $45^{\circ}$ counter-clockwise from $\left(u_{t}, u_{g}\right)$-frame. Note that, the

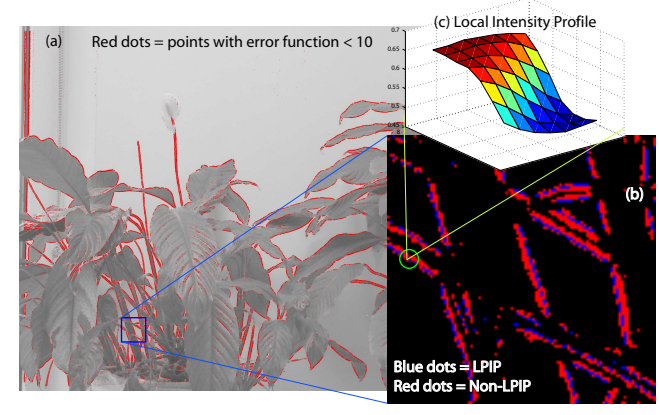

Figure 3. (a) Points detected by $E(R)<10$. (b) A magnified view showing the selected points being classifying into LPIP (blue) and non-LPIP (red), where LPIP is often surrounded by non-LPIP. (c) A local intensity profile of an LPIP

axis of the $\left(u_{\bar{t}}, u_{\bar{g}}\right)$-frame stays the farthest possible from the isophote and computing $\mathcal{G}_{1}$ on $\left(u_{\bar{t}}, u_{\bar{g}}\right)$-frame circumvents the above-mentioned singularity problem.

In practice, imposing a strict constraint equality as in Eq. 4 is unrealistic in the presence of derivative computation error due to image noise, quantization, and spatial discretization of an image. Therefore, an error function in Eq. 8 is used to choose the candidate points for LPIP. To simplify notation, we hereforth denote $R_{u_{\bar{t}}}$ as $R_{\bar{t}}, R_{u_{\bar{g}}}$ as $R_{\bar{g}}$ and so on.

$E(R)=\left|\frac{R_{\overline{t t}}}{R_{\bar{t}}^{2}}-\frac{R_{\overline{g g}}}{R_{\bar{g}}^{2}}\right|+\left|\frac{R_{\overline{t t}}}{R_{\bar{t}}^{2}}-\frac{R_{\bar{t} \bar{g}}}{R_{\bar{t}} R_{\bar{g}}}\right|+\left|\frac{R_{\overline{g g}}}{R_{\bar{g}}^{2}}-\frac{R_{\bar{t} \bar{g}}}{R_{\bar{t}} R_{\bar{g}}}\right|$

Our detection method using the above error function can detect LPIP points with small spatial support. In computer vision, image edge profile is often modeled by a 1D ramp, step or peak [11]. It is reasonable to find LPIP on a ramp edge profile, especially at the ramp center, and this hypothesis is empirically validated on an image shown Fig. 3. Fig. 3 (a) shows the points detected by $E(R)<10$. Note that, most of the points lies on image edges. In Fig. 3 (b), we further classify the detected points into the LPIP set and the non-LPIP set (formally defined in Sec. 4). Note that, LPIP's are mainly found at the middle of the edges and this supports the above-mentioned hypothesis.

For the work in [7], although the ramp edge profile in an irradiance image implies a uniform distribution of edge pixel value which is used as an assumption for their CRF estimation method. In constrast to our method, their method requires a larger support of ramp profile for constructing a reliable histogram and lacks a principled technique to detect image regions consistent with their physical assumption.

\subsection{Geometric Significance of Equality Constraint}

The derivative equality constraint specified in Eq.4 has an intuitive geometric interpretation. We first introduce three geometric quantities [4] called the isophote curvature $(\kappa)$, the normalized 2 nd-derivative in the gradient direction 


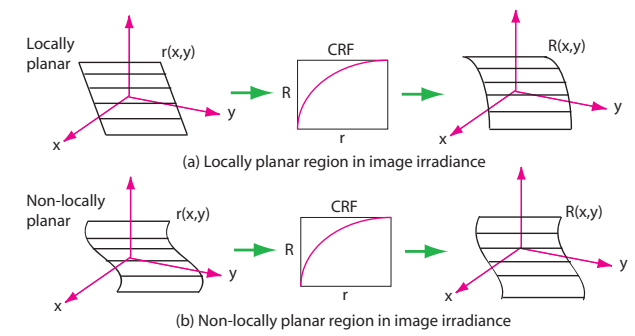

Figure 4. The CRF transformation preserves the shape of an isophote. (a) A LPIP region in image irradiance retains the linear isophotes in the intensity domain. (a) A non-LPIP region with linear isophote which satisfies the equality constraint.

$(\lambda)$, and the flow line curvature $(\mu)$, all expressed in the $\left(u_{t}, u_{g}\right)$-frame, as shown in Eq. 9.

$$
\kappa=-\frac{R_{t t}}{R_{g}}, \lambda=\frac{R_{g g}}{R_{g}} \text { and } \mu=-\frac{R_{t g}}{R_{g}}
$$

The basic meaning of a curve curvature is the local curve deviation from a tangent line. The isophote curvature $\kappa$ is the local curve deviation in the isophote direction, where $\kappa=0$ indicating a linear isophote. The flow line curvature $\mu$ is the local change in the gradient vector field along the isophote, where $\mu=0$ indicating same-shape isophotes along the gradient direction (see Fig. 4). Then, $\kappa=\mu=0$ if and only if a local region is composed of linear isophotes.

Proposition 1 (Decomposition of $\mathcal{G}_{1}$ ). The $\mathcal{G}_{1}$ can be decomposed as below:

$\frac{R_{\overline{t t}}}{R_{\bar{t}}^{2}}=\frac{\lambda-\kappa-2 \mu}{R_{g}}, \frac{R_{\overline{g g}}}{R_{\bar{g}}^{2}}=\frac{\lambda-\kappa+2 \mu}{R_{g}}$ and $\frac{R_{\bar{t} \bar{g}}}{R_{\bar{t}} R_{\bar{g}}}=\frac{\lambda+\kappa}{R_{g}}$

Proposition 1 associates $\mathcal{G}_{1}$ with three geometrically meaningful quantities. The decomposition immediately leads to Proposition 2 which puts an equivalence relationship between the equality constraint and the vanishing of the isophote curvature and the flowline curvature. This indicates a local geometric structure with linear isophotes, which resembles the local image intensity profile shown in Fig. 3 (c).

Proposition 2 (Geometric Significance of Equality Constraint in Eq.4).

$$
\left\{\frac{R_{\overline{t t}}}{R_{\bar{t}}^{2}}=\frac{R_{\overline{g g}}}{R_{\bar{g}}^{2}}=\frac{R_{\bar{t} \bar{g}}}{R_{\bar{t}} R_{\bar{g}}}\right\} \Longleftrightarrow\{\kappa=\mu=0\}
$$

and locally, $R=R\left(u_{g}\right)$, i.e., an arbitrary function depends only on $u_{g}$ in the gradient axis, with the linear isophote coincides with the tangent axis.

Proposition 2 indicates detection ambiguity by showing that the constraint equation detects points in a general region with linear isophotes, for which the LPIP set is only a subset. In other words, the constraint detects points on both regions of $f(a x+b y+c)$ and $f(h(a x+b y+c))$, where $h$ is an arbitrary function, as illustrated in Fig. 4. Hence, we propose a model-based inferencing method (Sec. 4) to further detect LPIP from the candidate set found by the error function (Eq. 8).

\subsection{Model based CRF Estimation}

Detection ambiguity motivates model-based CRF estimation method, as a restricted function space imposed by a CRF model makes CRF estimation more reliable in the presence of noise. Property 3 leads us to a suitable CRF model for our method.

Property 3 (Relationship with Gamma Curves). For gamma curves, $R=f(r)=r^{\gamma}, \mathcal{G}_{1}$ has a simple relationship with the parameter $\gamma$ :

$$
\mathcal{G}_{1}(R)=\left(\frac{\gamma-1}{\gamma R}\right) \text { and } \gamma=\frac{1}{1-\mathcal{G}_{1}(R) R} \doteq Q(R)
$$

Note that, estimating $\gamma$ from the $\mathcal{G}_{1}(R)$ expression in Eq. 12 will encounter a singularity at $R=0$. However, Eq. 12 suggests that when $\mathcal{G}_{1}(R)$ is transformed to $Q(R)$, $Q(R)$ is just equal to $\gamma$, a constant function independent of $R$, which is also bounded for concave gamma curves $(\gamma \in[0,1])$. Hence, Property 3 gives us a compatible pair of CRF model and an expression $Q(R)$ for estimating CRF.

\subsubsection{Generalized Gamma Curve Model}

However, gamma curves are limited in representing realworld CRF's. Therefore, we propose a generalized gamma curve model (GGCM) which has a good fit to the real-world CRF's in the DoRF database [5]. GGCM provides two representations of CRF with $f: r \mapsto R$ (GGCM $f$ ) and $g: R \mapsto r(\mathrm{GGCM} g)$, as shown in Eq. 13.

$$
f(r)=r^{P(r, \tilde{\alpha})} \text { and } g(R)=R^{1 / P(R, \tilde{\alpha})}
$$

where $\tilde{\alpha}=\left[\alpha_{1}, \ldots, \alpha_{n}\right], P(x, \tilde{\alpha})=\sum_{i=0}^{n} \alpha_{i} x^{i}$ is a $n$-th order polynomial, with $n+1$ parameters. Note that, GGCM is reduced to the gamma curve model when the polynomial is reduced to a constant term. Additionally, CRF is commonly represented by a function $f$ with $f(0)=0$ and $f(1)=1$. As $r$ can only be recovered with precision up to a linear scaling and an offset of the actual image irradiance, it is reasonable to normalize $r$ to $[0,1]$.

Various CRF model has been proposed for CRF estimation. One of the earliest model, $f(r)=\alpha+\beta r^{\gamma}$, is borrowed from the photographic emulsion response function [8], essentially a gamma curve after normalization. A general polynomial CRF model is then proposed in [10]. Recently, an empirical EMOR model [5] is obtained from performing principle component analysis (PCA) on 201 real-world CRF's. As the empirical model lacks the differentiable property of an analytic model, it is not suitable for our method. We evaluate GGCM by performing least square fit of the model to the 201 real-world CRF's 
Table 1. Mean RMSE $\left(\times 10^{-2}\right)$ of the proposed CRF model

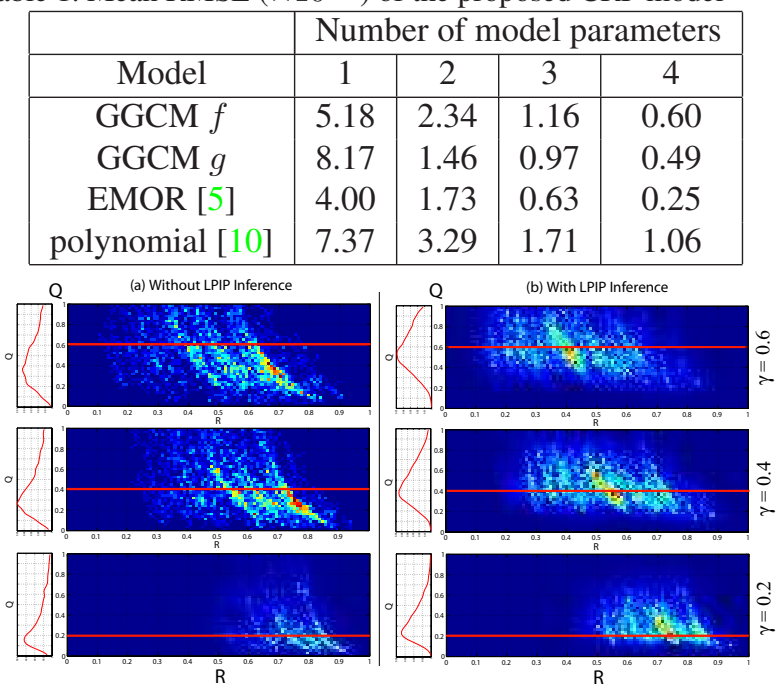

Figure 5. The typical $Q-R$ histogram of LISO from single gammacurve simulation images with $\gamma=0.2,0.4$ and 0.6 for (a) without LPIP inference and (b) with LPIP inference. The red curve on the left is the marginal $Q$ distribution. The red line in each graph indicates the ground truth value of $\gamma$

in the DoRF database. The goodness of fit for each CRF is measured by RMSE and is shown in Table $1^{1}$. Note that GGCM performs slightly worse than the empirical EMOR [5] model but outperforms the polynomial CRF model [10], which is a commonly used analytic CRF model.

\section{Addressing Detection Ambiguity Issues}

Due to detection ambiguity, the equality constraint detects locally linear isophote points (LISO), for which LPIP is a subset. As our algorithm requires LPIP for CRF estimation, we in this section present a data-driven approach to infer how likely an LISO to be an LPIP, by exploring the common characteristics of LPIP in terms of their derivative quantities and their spatial layout shown in Fig. 3.

To study the effect of detection ambiguity, we generate a set of simulation images by transforming more than 10 irradiance images (extracted from RAW format images which are the direct output from a CCD image sensor) with gamma curves $f(r)=r^{\gamma}$ for $\gamma=0.2,0.4$ and 0.6. We observe that more than $92 \%$ of the real-world digital camera CRF's in the DoRF database lie in between $r^{0.2}$ and $r^{0.6}$. We define the LISO set $\mathcal{S}_{L I S O}$ as:

$$
\mathcal{S}_{\text {LISO }}=\{(x, y): E(R(x, y))<10\}
$$

Fig. 5 (a) shows a typical distribution of the detected LISO points in $Q-R$ space. Note that, from Property 3, the $Q$ function corresponding to gamma curves is a constant. From the marginal $Q$ distribution on the left, we see that as

\footnotetext{
${ }^{1}$ The mean RMSE for the EMOR and the polynomial CRF model are extracted from [5].
}

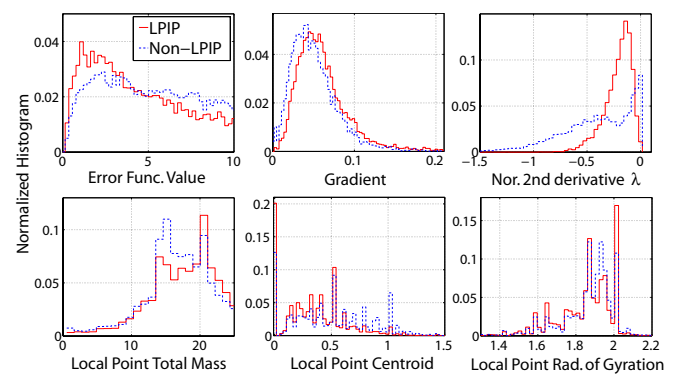

Figure 6. The class-dependent feature distributions.

$\gamma$ increases from 0.2 to 0.6 , the $Q$ distribution density consistently shifts to a higher value, as predicted by the theory. However, the mode of the distribution does not coincide exactly with the ground-truth value of $\gamma$ and this is an effect of detection ambiguity, which we will rectify through LPIP inference.

For LPIP inference, we define two groups of features. The first group consists geometric quantities related to the selection of LISO and the computation of $Q$, i.e., $E(R)$ value, gradient value, and the value of the normalized 2ndderivative in the gradient direction ( $\lambda$, see Equ. 9). These features are for capturing the geometric difference between LPIP and non-LPIP. The second group consists of the moment features for capturing the specific spatial layout of LPIP in the binary LISO map $b(x, y)$, where $b(x, y)=1$ if $(x, y) \in \mathcal{S}_{L I S O}$, and $b(x, y)=0$ otherwise. Specifically, we compute the 1 st to 3 rd moment quantities on $b(x, y)$, i.e., the total mass $m_{0}=\sum_{W_{5 \times 5}} b(x, y)$, the centriod $m_{1}$, and the radius of gyration $m_{2}$ (Eq. 16), in $5 \times 5$ local windows $W_{5 \times 5}$.

$$
\begin{aligned}
& \left(\begin{array}{ll}
m_{1}^{x} & m_{2}^{x} \\
m_{1}^{y} & m_{2}^{y}
\end{array}\right)=\frac{1}{m_{0}} \sum_{(x, y) \in W_{5 \times 5}}\left(\begin{array}{ll}
x & x^{2} \\
y & y^{2}
\end{array}\right) b(x, y) \\
& m_{1}=\sqrt{\left(m_{1}^{x}\right)^{2}+\left(m_{1}^{y}\right)^{2}} \text { and } m_{2}=\sqrt{m_{2}^{x}+m_{2}^{y}}
\end{aligned}
$$

For the class-dependent feature distributions, we define the LPIP set $\mathcal{S}_{L P I P}$, and the non-LPIP set $\mathcal{S}_{n o n-L P I P}$ on the simulation images as below:

$$
\mathcal{S}_{L P I P}=\left\{(x, y):|Q(x, y)-\gamma| \leq 0.1,(x, y) \in \mathcal{S}_{L I S O}\right\}
$$

$\mathcal{S}_{\text {non-LPIP }}=\left\{(x, y):|Q(x, y)-\gamma|>0.1,(x, y) \in \mathcal{S}_{L I S O}\right\}$

The former condition chooses the points that satisfy the derivative equality constraint (thus in $\mathcal{S}_{L I S O}$ ) and their $Q$ value are close to the ground-truth value of $\gamma$. Only LPIP points meet these conditions simultaneously. The latter condition specifies points in the LISO set but having $Q$ values distant from the ground-truth $\gamma$, thus corresponding to the non-LPIP points. Fig. 6 shows the class-dependent feature distribution for $\mathcal{S}_{L P I P}$ and $\mathcal{S}_{n o n-L P I P}$. Note that, $\mathcal{S}_{L P I P}$ dominates the low $E(R)$ value, as expected by the theory. For the normalized 2nd-derivative feature $\lambda$, the high and 


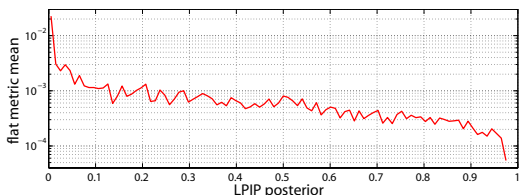

Figure 7. Relation between LPIP posterior and the flatness measurement in an irradiance image. Note the log scale on the y-axis.

low values are dominated by $\mathcal{S}_{\text {non-LPIP }}$, and this is related to the spatial structure of $\mathcal{S}_{\text {non-LPIP }}$ and the higher computational error for the 2nd-derivatives. For the gradient feature, $\mathcal{S}_{\text {non-LPIP }}$ dominates at the low values, as regions with low gradient (i.e., more horizontal) tend to suffer more from the quantization noise. On the other hand, the distribution of the moment features can be explained by the spatial structure of $\mathcal{S}_{L P I P}$ as shown in Fig. 3 (b).

For LPIP inference, we adopt Bayesian approach and enforce an independence assumption on the features $f_{i}$ :

$$
P(\tilde{f} \mid c)=\prod_{i=1}^{N} P\left(f_{i} \mid c\right) \text { where } \tilde{f}=\left[f_{1}, \ldots, f_{N}\right]
$$

where $c \in\left\{\mathcal{S}_{L P I P}, \mathcal{S}_{\text {non-LPIP }}\right\}$. Feature independence is crucial so that the inference does not capture the specificity of the gamma curves from the geometric features and hence is generalizable to more complex CRF curves than gamma curves. The a-posterior probability of an LISO being an LPIP is given by:

$$
P(c \mid \tilde{f})=\frac{P(\tilde{f} \mid c) P(c)}{P(\tilde{f})}=\frac{P(\tilde{f} \mid c) P(c)}{\sum_{c} P(\tilde{f} \mid c) P(c)}
$$

where $P(c)$ is the ratio of points belonging to the class $c$.

Fig. 5 (b) shows the distribution of LISO in $Q-R$ space, after incorporating the LPIP posterior as weights. It is obvious from the marginal $Q$ distribution that the mode of the distributions coincides very closely to the ground-truth gamma value $\gamma$, a sign of overcoming the effect of detection ambiguity. To further validate the effectiveness of LPIP inference, we measure the local planarity of an irradiance image using a simple metric, $m_{f}=\left(r_{x x}^{2}+r_{y y}^{2}\right)^{0.5}$, where for a plane, $m_{f}=0$. Fig. 7 shows that $m_{f}$ decreases (i.e., more planar) as the LPIP posterior increases, and hence vouches for the effectiveness of the proposed Bayesian method in selecting the true LPIP points.

\section{CRF Estimation}

\subsection{Objective Function for CRF Estimation}

Eq. 4 expresses $\mathcal{G}_{1}$ as a functional of $f$. Given $r=g(R)$ and $g=f^{-1}$, we can also express $\mathcal{G}_{1}$ in terms of $g$ as in Eq. 21. In this paper, we estimate CRF using $g(R)=$ $R^{\left(1 /\left(\alpha_{0}+\alpha_{1} R\right)\right)}$, whose $Q(R)$ is given by Eq. 22 .

$$
\mathcal{G}_{1}(R)=\frac{f^{\prime \prime}(r)}{f^{\prime}(r)^{2}}=-\frac{g^{\prime \prime}(R)}{g^{\prime}(R)}, \quad Q(R)=\frac{g^{\prime}(R)}{1+g^{\prime \prime}(R) R}
$$

$$
\begin{gathered}
Q(R)=\frac{\left(\alpha_{0}+\alpha_{1} R\right)^{2}\left(\alpha_{1} \ln (R)-\alpha_{0}+\alpha_{1} R\right)}{T} \\
T=\alpha_{0}^{2}+\alpha_{0} \alpha_{1} R\left(\alpha_{0}(\ln (R)+1)-2(1-\ln (R))\right)+ \\
\left(\alpha_{1} R\right)^{2}\left(1-4 \alpha_{0}-2 \alpha_{1} R+(\ln (R)-2)\left(\alpha_{1} R+\ln (R)\right)\right)
\end{gathered}
$$

We fit $Q(R)$ to the computed data $\left\{\left(Q^{n}, R^{n}\right)\right\}_{n=1}^{N}$ over N detected LISO points by minimizing the objective function in Eq. 24. Note that the best CRF parameter $\tilde{\alpha}^{*}$ is estimated by a weighted least-square criterion, where the weight is the conditional histogram of $Q$ given $R$. The reason for the conditional weight is to prevent the optimization from being dominated by the data on some specific $R$ which happen to be found abundant on an image.

$$
\tilde{\alpha}^{*}=\arg \min _{\tilde{\alpha}} \sum_{j, k} P\left(Q_{j} \mid R_{k}\right)\left|Q_{j}-Q\left(R_{k}, \tilde{\alpha}\right)\right|^{2}
$$

where $\tilde{\alpha}=\left(\alpha_{0}, \alpha_{1}\right), Q_{j}$ and $R_{k}$ are respectively the discrete samples on $Q$ and $R$ (representing the histogram bin centers), $P\left(Q_{j} \mid R_{k}\right)=P\left(Q_{j}, R_{k}\right) / P\left(R_{k}\right)$, and

$$
P\left(Q_{j}, R_{k}\right)=\sum_{n=1}^{N} p\left(\mathcal{S}_{L P I P} \mid \tilde{f}^{n}\right) \mathbf{1}_{\left[Q_{j}, R_{k}\right]}\left\{\left(Q^{n}, R^{n}\right)\right\}
$$

where $p\left(\mathcal{S}_{L P I P} \mid \tilde{f}^{n}\right)$ is given in Eq. $20,\left[Q_{j}, R_{k}\right]$ is the bin corresponding to the bin center $\left(Q_{j}, R_{k}\right)$, and the indicator function, $\mathbf{1}_{A}\{a\}=1$ if $a \in A$.

\subsection{Joint Estimation for Multiple-channel Images}

Apart from single-channel images, the proposed method can also be applied to RGB images. Joint estimation of the RGB CRF's can be performed by constraining the similarity between the RGB CRF's, as in Eq. 26 with $\tilde{\alpha}=$ $\left\{\tilde{\alpha}_{r}, \tilde{\alpha}_{g}, \tilde{\alpha}_{b}\right\}$. In practice, the RGB CRF's of a camera are quite similar.

$$
\begin{aligned}
& \tilde{\alpha}^{*}=\arg \min _{\tilde{\alpha}}\left\{\sum_{j, k, c} P\left(Q_{j} \mid R_{k}\right)\left|Q_{j}-Q\left(R_{k}, \tilde{\alpha}_{c}\right)\right|^{2}\right. \\
& \left.+\sum_{c_{1}>c_{2}}\left(\frac{1}{K} \sum_{k=1}^{K}\left(g\left(R_{k}, \tilde{\alpha}_{c_{1}}\right)-g\left(R_{k}, \tilde{\alpha}_{c_{2}}\right)\right)^{2}\right)^{\frac{1}{2}}\right\}
\end{aligned}
$$

Furthermore, if we have $M$ single-channel images with the same CRF, we can average up their conditional histograms to increase the data coverage on $R$ (R-coverage), as in Eq. 27, and then form an objective function as in Eq. 24.

$$
P\left(Q_{j} \mid R_{k}\right)=\frac{1}{M} \sum_{m=1}^{M} P_{m}\left(Q_{j} \mid R_{k}\right)
$$

\section{Implementation Aspects of the Algorithm}

\subsection{Computation of Image Derivative}

There is a huge literature on techniques for computing image derivatives, which includes finite difference, Gaussian scale-space derivatives, and many more. These methods in general work well for common applications (e.g. 
Table 2. Overall RMSE $\left(\times 10^{-2}\right)$ for CRF Estimation

\begin{tabular}{|c|c|c|c|c|c|}
\hline Stat. & $E_{1}$ & $E_{r g b}$ & $E_{2}$ & $E_{3}$ & $E_{4}$ \\
\hline Mean & 2.91 & 2.66 & 2.13 & 1.90 & 1.76 \\
2nd Mom & 3.43 & 2.95 & 2.41 & 2.10 & 1.94 \\
\hline
\end{tabular}

edge detection). However, our differential method involves computation of derivative ratios from digital images which requires specialized techniques to ensure the computational accuracy and robustness to image noise. There are prior works that involves computing derivative ratios from digital images, such as the works on curve invariants [12] and edge curvatures [11], which use local polynomial fitting method [11,9] for computing derivative and achieves good accuracy in derivative estimation. This method presets the derivative kernel size, and hence has limited adaptability to the wide range of scales in an image. In our work, we use cubic smoothing B-spline [1] with $\mathbb{C}^{2}$ continuity for computing image derivatives. B-spline is a function consists of local piecewise polynomials with a global continuity property. Cubic smoothing B-spline is obtained by simultaneously minimizing a data fitting energy and an $\mathbb{L}^{2}$ norm on the second-order partial derivatives (producing smoothing effort). Comparing to local polynomial fitting, we find that cubic smoothing B-spline is more adaptive in terms of image scales and therefore producing considerably more accurate image derivatives.

\subsection{Error Metric Calibration}

Although $Q(R)$ is compatible with GGCM for CRF estimation through curve-fitting, the space of $Q(R)$ is not 'flat', i.e., its metric is dependent on the CRF curve parameter. For example, RMSE between $r^{0.1}$ and $r^{0.2}$ is 0.0465 , and that between $r^{0.5}$ and $r^{0.6}$ is 0.0771 , almost twice of the former, while their $Q(R)$ RMSE are the same. Note that, for gamma curves, RMSE between two $Q(R)$ is simply $\left|\gamma_{1}-\gamma_{2}\right|$. In a non-flat space, curve-fitting performance biases towards certain CRF. Interestingly, the error metric calibration can be formulated as a problem of reparametrizing a space curve with its arc length and leads to Proposition 3.

Proposition 3 (Error metric calibrated). The error metric in the $Q(R)$ space can be calibrated with respect to gamma curves, $f(r)=r^{\gamma}$, by a transform on $Q$ :

$$
\bar{Q}=\frac{\sqrt{3}}{\sqrt{3}-1}\left(1-\sqrt{\frac{1}{2 Q+1}}\right)
$$

Such calibrated metric can then be used to replace that in Eq. 24 to improve the estimation accuracy.

\section{Experiments}

We test our CRF estimation method using 20 uncompressed RGB-color images (i.e., 60 single-channel images
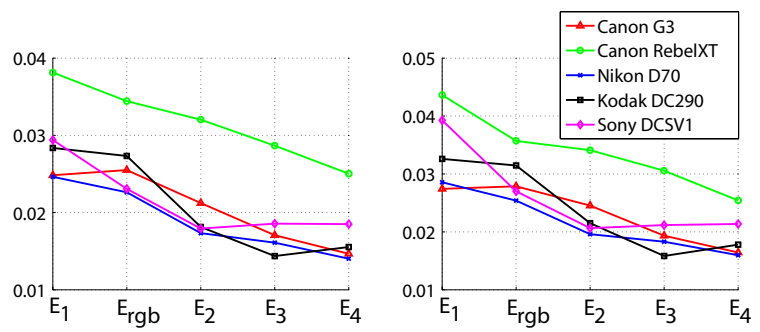

Figure 8. The plot of RMSE mean (Left) and RMSE 2nd-moment (Right) for different cameras and different CRF estimation strategies $E_{1}$ to $E_{4}$ and $E_{r g b}$.

cropped to the size of $1500 \times 2000$ pixel without filtering or subsampling) from each of the five models of camera, i.e., Canon G3, Canon RebelXT, Nikon D70, Kodak DC290, Sony DSCV1 (a total of 100 RGB-images from four major camera manufacturers). We select images with at least $80 \%$ data coverage in $R$ ( $R$-coverage) so that a proper curve fitting is possible in the $R-Q$ space.

We estimate the ground-truth CRF for the cameras using Macbeth chart (using multiple images with different exposures). For each camera, the ground-truth CRF's are indeed similar over RGB channels, with the averaged inter-colorchannel CRF difference measured in RMSE being 0.0161. We test our methods using single-color-channel images (denoted as $E_{1}$ ), RGB images (denoted as $E_{r g b}$ ), and also combinations of 2 to 4 single-color-channel images from the same camera (denoted by $E_{2}$ to $E_{4}$ ). The discrepancy between the estimated and the ground-truth CRF is measured by RMSE. The mean RMSE (measuring accuracy) and the 2nd-moment of RMSE (measuring stability) for the five cameras over RGB color channels and all images of a camera is shown in Fig. 8. The overall RMSE mean and RMSE 2nd-moment (over all cameras) are shown in Table 2. Note that, both estimation accuracy and stability improve as more images are available, which verifies the importance of Rcoverage as combining conditional histograms strictly increases R-coverage.

Fig. 8 shows that the estimated CRF's for Canon RebelXT have the least accuracy and stability. As shown in Fig. 9, the estimated CRF's for Canon RebelXT deviate slightly from the knee of the groundtruth curve. Note that, the knee of the Canon RebelXT CRF is very close to linear, and our method does not perform well on linear CRF (due to the basic principle of our method which relies on locally planar regions in an irradiance image). Fig. 9 (a) and (b) respectively show the estimated blue-color channel CRF's for the five models of camera with $E_{1}$, and $E_{4}$. The estimation results for other color channels are similar. Among all, the CRF of Canon RebelXT and Nikon D70 have the largest difference with a RMSE of 0.0781 (averaged over RGB). For a side-by-side comparision, the estimated bluecolor channel CRF's for Canon RebelXT and Nikon D70 with $E_{1}$ and $E_{4}$ are shown in the lower-right plot in Fig. 9. 


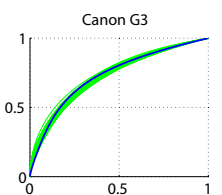

Kodak DC290
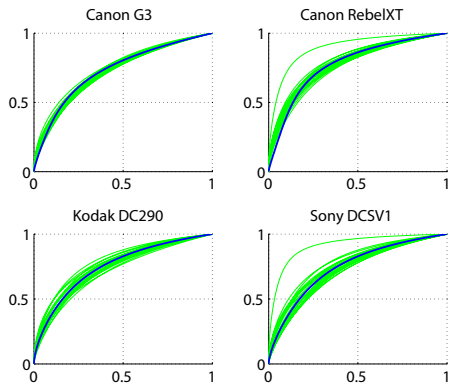

Sony DCSV1
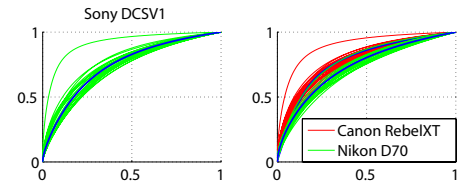

(a) Estimated CRF's with $\mathrm{E}_{1}$

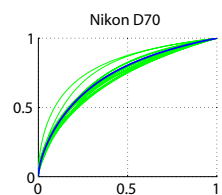

0.5

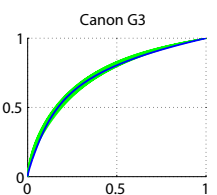

Kodak DC290

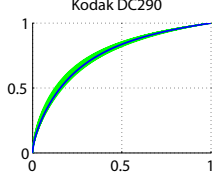

(b) Estimated CRF's with $\mathrm{E}_{4}$

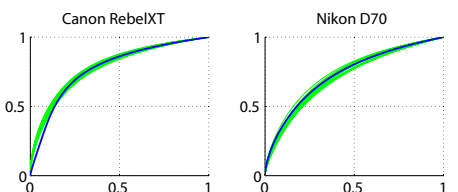

Sony DCSV1

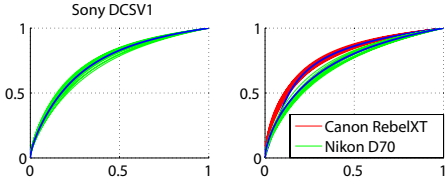

0.5

Figure 9. Estimated blue-color channel CRF's for the five models of camera using (a) a single color-channel image ( $\left.E_{1}\right)$ and (b) four blue-color-channel images $\left(E_{4}\right)$. The thick blue line represents the ground-truth CRF. The CRF of Canon RebelXT and Nikon D70 are most different and the estimated CRF for Canon RebelXT and Nikon D70 are shown in the bottom-right plots.
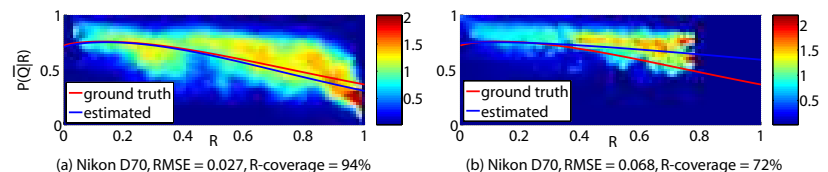

Figure 10. Curve-fitting in $\bar{Q} \times R$ space with data of (a) high Rcoverage, (b) Low R-coverage. The thick blue line represents the ground-truth $Q(R)$ curve.

Note that, a slight confusion of the estimated CRF's of the two cameras is observed for $E_{1}$, which is gradually cleared for $E_{4}$.

Two examples of curve-fitting respectively on data of high and low R-coverage are shown in Fig. 10. Note the importance of the end-point data in $R \in[0,1]$ which can be seen as the boundary condition for accurate CRF estimation. As experiments in [7] are only conducted on two cameras (different from ours) and the test grayscale images of the digital camera are converted from RGB images (instead of using single-color-channel images), rigorous performance comparison is not possible.

\section{Conclusions}

In this paper, we presented a geometry invariant-based method for estimating CRF. In contrast to the single-image CRF estimation methods in the prior works which lack a principled technique to select data consistent to their inherent assumption, our method provides a constraint equation for selecting the potential locally planar irradiance points. Comparing to the prior works [7], our experiment was conducted over more extensive data and our method is flexible in that we can increase its estimation accuracy and stability when more than one image is available. The geometry invariance theory is novel and may be of wide interest. Techniques in our implementation such as smoothing B-spline for computing image derivatives and the procedure for calibrating the error metric may be useful for other applications. Currently, the algorithm is for the 1st-order geometry invariants, the next step would be to develop an algorithm for the 2nd-order geometry invariants.

\section{Acknowledgment}

This work has been supported in part by NSF Cyber Trust program (IIS-04-30258). Thanks to Singapore A*STAR for supporting the first author.

\section{References}

[1] C. de Boor. A Practical Guide to Splines. Springer-Verlag, New York, 1978. 7

[2] P. E. Debevec and J. Malik. Recovering high dynamic range radiance maps from photographs. In ACM SIGGRAPH, pages 369-378, 1997. 1, 2

[3] H. Farid. Blind inverse gamma correction. IEEE Trans. Image Proc., 10(10):1428-1433, 2001. 2

[4] L. M. J. Florack, B. M. ter Haar Romeny, J. J. Koenderink, and M. A. Viergever. Scale and the differential structure of images. Image Vision Comput., 10(6):376-388, 1992. 3

[5] M. Grossberg and S. Nayar. What is the space of camera response functions? In IEEE CVPR, pages 602-9, 2003. 2, 4, 5

[6] S. Lin, J. Gu, S. Yamazaki, and H.-Y. Shum. Radiometric calibration from a single image. In IEEE CVPR, volume 2, pages 938-945, June 2004. 1, 2

[7] S. Lin and L. Zhang. Determining the radiometric response function from a single grayscale image. In IEEE CVPR, volume 2, pages 66-73, June 2005. 1, 2, 3, 8

[8] S. Mann. Comparametric equations with practical applications in quantigraphic image processing. IEEE Trans. Image Proc., 9(8):1389-1406, 2000. 1, 2, 4

[9] P. Meer and I. Weiss. Smoothed differentiation filters for images. In ICPR, pages 121-126, 1990. 7

[10] T. Mitsunaga and S. Nayar. Radiometric self calibration. In IEEE CVPR, pages 374-380, 1999. 1, 2, 4, 5

[11] T. Vieville and O. D. Faugeras. Robust and fast computation of unbiased intensity derivatives in images. In $E C C V$, pages 203-211, 1992. 3, 7

[12] I. Weiss. Noise-resistant invariants of curves. IEEE Transactions on Pattern Analysis and Machine Intelligence, 15(9):943-948, 1993. 7

[13] F. Xiao, J. Farrell, J. DiCarlo, and B. Wandell. Preferred color spaces for white balancing. In SPIE Electronic Imaging Conference, volume 5017, 2003. 2 